\title{
POSITIVE DEFINITENESS ON PRODUCTS VIA GENERALIZED STIELTJES AND OTHER FUNCTIONS
}

\author{
V. A. Menegatto
}

Abstract. Let $(X, \rho)$ and $(Y, \sigma)$ be quasi-metric spaces and $\lambda$ a fixed positive real number. This paper establishes the positive definiteness of functions of the form

$$
G_{r}(t, u)=\frac{1}{h(u)^{r}} f\left(\frac{g(t)}{h(u)}\right),(t, u) \in\left\{\rho\left(x, x^{\prime}\right): x, x^{\prime} \in X\right\} \times\left\{\sigma\left(y, y^{\prime}\right): y, y^{\prime} \in Y\right\},
$$

on $X \times Y$, where $r \geqslant \lambda, f$ belongs to the convex cone of all generalized Stieltjes functions of order $\lambda$, and $g$ and $h$ are positive valued conditionally negative definite functions on $(X, \rho)$ and $(Y, \sigma)$, respectively. As a bypass, it establishes the positive definiteness of functions of the form

$$
H_{r}(t, u)=\frac{1}{g(t)^{r}} f\left(\frac{g(t)}{h(u)}\right),(t, u) \in\left\{\rho\left(x, x^{\prime}\right): x, x^{\prime} \in X\right\} \times\left\{\sigma\left(y, y^{\prime}\right): y, y^{\prime} \in Y\right\},
$$

for a generalized complete Bernstein function $f$ of order $\lambda$, under the same assumptions on $r, g$ and $h$. The paper also provides necessary and sufficient conditions for the strict positive definiteness of the two models when the spaces involved are metric. The two results yield additional methods to construct positive definite and strictly positive definite functions on a product of metric spaces by integral transforms.

Mathematics subject classification (2010): 42A82, 43A35.

Keywords and phrases: Positive definite functions, generalized Stieltjes functions, conditionally negative definite functions, Gneiting's class, complete Bernstein functions.

\section{REFERENCES}

[1] A. Belton, D. Guillot, A. Khare, and M. Putinar, A Panorama of Positivity. I: Dimension Free, In: Aleman A., Hedenmalm H., Khavinson D., Putinar M. (eds). Analysis of Operators on Function Spaces. Trends in Mathematics, Birkhäuser, Cham, 117-165, 2019.

[2] C. Berg, J. P. R. Christensen, AND P. Ressel, Harmonic analysis on semigroups. Theory of positive definite and related functions, Graduate Texts in Mathematics, 100, Springer-Verlag, New York, 1984.

[3] L. Bondesson, A general result on infinite divisibility, Ann. Probab. 7, 6 (1979), 965-979.

[4] T. Gneiting, Nonseparable, stationary covariance functions for space-time data, J. Amer. Stat. Assoc. 97, 458 (2002), 590-600.

[5] R. A. Horn And C. R. Johnson, Matrix analysis. Second edition, Cambridge University Press, Cambridge, 2013.

[6] Y. Kapil, R. Pal, A. Aggarwal, AND M. Singh, Conditionally negative definite functions, Mediterr. J. Math. 15, Art. 199 (2018), 1-12.

[7] D. KarP AND E. PRILEPKInA, Generalized Stieltjes functions and their exact order, J. Class. Anal. 1, 1 (2012), 53-74.

[8] S. Koumandos And H. L. Pedersen, On asymptotic expansions of generalized Stieltjes functions, Comput. Methods Funct. Theory 15, 1 (2015), 93-115.

[9] S. Koumandos And H. L. Pedersen, On generalized Stieltjes Functions, Constr. Approx. 50, 1 (2019), 129-144. 
[10] V. A. Menegatto, Positive definite functions on products of metric spaces via generalized Stieltjes functions, Proc. Amer. Math. Soc. 148, 11 (2020), 4781-4795.

[11] V. A. Menegatto, Strictly positive definite kernels on the Hilbert sphere, Appl. Anal. 55, 1-2 (1994), 91-101.

[12] V. A. Menegatto, C. P. Oliveira, and E. Porcu, Gneiting class, semi-metric spaces, and isometric embeddings, Constr. Math. Anal. 3, 2 (2020), 85-95.

[13] J. von Neumann and I. J. Schoenberg, Fourier integrals and metric geometry, Trans. Amer. Math. Soc. 50, 0 (1941), 226-251.

[14] R. REAMS, Hadamard inverses, square roots and products of almost semidefinite matrices, Linear Algebra Appl. 288, 1-3 (1999), 35-43.

[15] R. L. Schilling, R. Song, And Z. Vondracek, Bernstein functions. Theory and applications, Second edition, De Gruyter Studies in Mathematics 37, Walter de Gruyter \& Co., Berlin, 2012.

[16] A. D. Sokal, Real-variables characterization of generalized Stieltjes functions, Expo. Math. 28, 2 (2010), 179-185.

[17] J. H. Wells AND L. R. WiLliams, Embeddings and extensions in analysis, Ergebnisse der Mathematik und ihrer Grenzgebiete, Band 84, Springer-Verlag, New York-Heidelberg, 1975.

[18] P. White AND E. PorCU, Towards a complete picture of covariance functions on spheres cross time, Electron. J. Stat. 13, 2 (2019), 2566-2594.

[19] V. P. Zastavnyi And E. Porcu, Characterization theorems for the Gneiting class of space-time covariances, Bernoulli 17, 1 (2011), 456-465. 